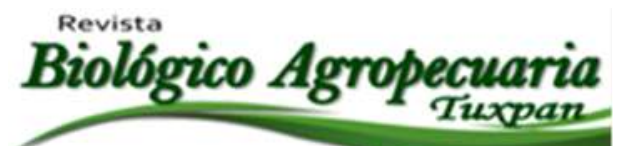

\title{
Variaciones analizadas de la producción de huizache (Acacia farnesiana) en una población nativa versus una plantación urbana de la región centro sur de Chihuahua
}

Variations analyzed of the production of huizache (Acacia farnesiana) in a native population versus an urban plantation of the central south region of Chihuahua

Magaña Magaña José Eduardo ${ }^{1 凶}$, Romero Lara María de los Ángeles ${ }^{2}$.Victor Hugo Villarreal Ramirez ${ }^{3}$, Luisa Patricia Uranga Valencia ${ }^{4}$, Christian Mauricio Kiessling Davison ${ }^{5}$

Profesor de la Facultad de Ciencias Agrícolas y Forestales de la Universidad Autonoma de Chihuahua 1,3,4,5,

\Autor para correspondencia: emagana@uach.mx

Recibido: 05/08/2019

Aceptado: 23/12/2019

\section{RESUMEN}

La investigación se realizó en una población nativa de huizache de Naica, Saucillo y una plantación urbana de Delicias, Chihuahua. En el ecosistema nativo se realizó un estudio exploratorio con tres tratamientos $(10 \%, 15 \%, 20 \%)$ de una hormona y un testigo, para determinar la altura de aplicación del fuste principal que fue desde $50 \mathrm{~cm}$ y la distancia de corte de las ramas secundarias desde su bifurcación que fue de $15 \mathrm{~cm}$. La longitud de corte fue de $2.5 \mathrm{~cm}$ y la profundidad de $0.5 \mathrm{~cm}$. Se observó que las plantas no manifestaron signos externos en el follaje y en la producción de flores y vainas en el ciclo siguiente. Se realizó como segunda fase, un experimento de bloques completamente al azar con los tres tratamientos de la hormona vegetal con seis repeticiones. Los resultados promedio por tratamiento indicaron que el $20 \%$ fue el mejor tratamiento con 170.97 gr. de goma durante dos meses. En la plantación urbana de una hectárea de huizache, se aplicó el mismo diseño experimental evaluando los mismos tratamientos. Los resultados obtenidos demuestran que el mejor tratamiento en promedio fue el de $10 \%$ con 374.14 gr. de goma durante tres meses.

Palabras clave: Etileno, Resina, Emulsificante, Planta del desierto, Industria alimenticia.

\begin{abstract}
The research was conducted in a native population of huizache of Naica, Saucillo and an urban plantation of Delicias, Chihuahua. In the native ecosystem was conducted an exploratory study with three treatments $(10 \%, 15 \%, 20 \%)$ applying a vegetative hormone and a witness, to determine the height of cut in the main trunk which was $50 \mathrm{~cm}$ and the distance of the secondary branches cut which was $15 \mathrm{~cm}$. The cutting length was $2.5 \mathrm{~cm}$ and depth of $0.5 \mathrm{~cm}$. It was noted that the plants did not manifest outward signs in the foliage and the production of flowers and pods in the next cycle. It was as a second phase, an experiment in completely randomized blocks with three treatments of the plant hormone with six replications. The average treatment results indicated that $20 \%$ was the best treatment with 170.97 gr. of
\end{abstract}


gum for two months. The same experimental design was applied in a urban planting of one hectare of huizache, it was applied the same experimental design evaluating the same treatments. The results obtained show that the best treatment on average was $10 \%$ with 374.14 gr. of gum for three months.

Keywords: Ethylene, Resin, Emulsifier, Desert plant, Food industry.

\section{INTRODUCCIÓN}

El Desierto Chihuahuense es considerado el más grande en Norteamérica y una de las regiones secas con mayor número de especies del mundo (Sutton, 2000). El propósito del presente trabajo de investigación fue el de contribuir al desarrollo económico de las comunidades rurales marginadas y de pobreza extrema que viven en el desierto de Chihuahua a través del aprovechamiento integral y sustentable de sus recursos naturales como el huizache y mezquite que conducen a preservar la biodiversidad. De acuerdo a la ONU (2016), la degradación de las zonas áridas en el mundo ha sido el resultado de las acciones del ser humano y están promoviendo retos más grandes para la lucha contra la pobreza de millones de seres humanos. Así mismo, la biodiversidad se perjudica por lo que se requiere concientizar en el cuidado y preservación de los ecosistemas, su mejora y el desarrollo social comunitario.

Las gomas son secreciones resinosas que producen algunas especies vegetales como un mecanismo de defensa natural, con el objetivo de proteger al árbol contra plagas y enfermedades (Verbeken et al., 2003). Las principales especies productoras de goma de mayor uso y demanda en la industria son las Acacia senegal y Acacia seyal de origen africano (Cabañas, 2012). La goma o resina de Acacia farnesiana puede sustituir a la goma arábiga que es usada como conservador natural de alimentos reduciendo así su importación (Reséndiz et al., 2016).
Las exportaciones africanas de la goma arábiga son provenientes de Acacia senegal y Acacia seyal. En crudo han ido aumentando en los últimos 25 años alcanzando en promedio anual de 35,000 t en 1992-1994 a 102,000 t en 20142016, lo equivalente en dólares de Estados Unidos de 95.4 millones de dólares a 150.3 millones de dólares aumentando en un $158 \%$. De igual forma la goma arábiga procesada paso de $17,000 \mathrm{t}$ a 53,000 t con valor de 74.4 millones de dólares a 192 millones de dólares en los mismos periodos.

Los principales países exportadores de goma arábiga (Acacia senegal y Acacia seyal) del mundo son Sudan con $66 \%$, el Chad con el $13 \%$ y Nigeria con el $8.5 \%$. Otros países que han aumentado su exportación son el Mali, Senegal y Camerún mientras que en Sudán del Sur, Etiopía y Kenia no se aprovecha completamente este recurso. Los principales importadores de la goma arábiga en crudo con el $75 \%$ son Francia e India, siendo Nexira (fabricante europeo), quien controla a nivel mundial el $50 \%$ de este (Conferencia de la Naciones Unidas sobre comercio y desarrollo, 2018).

La presente investigación tiene como objetivo general: producir goma de huizache en una población nativa y plantación urbana utilizando etileno en la localidad de Naica, Saucillo y ciudad Delicias, Chihuahua y como específicos: Definir cuál técnica o tratamiento es mejor para la producción de la goma de huizache sin que cause daño o deterioro al árbol; evaluar el comportamiento de las variables críticas de la población nativa en Naica, Saucillo y en plantación en Delicias, Chihuahua; producción de goma en base a los 
tratamientos aplicados y verificar que no exista daño biológico o infestación de plagas; realizar un análisis proximal de la goma de mezquite y de huizache (acacia spp) y compararlo con la goma arábiga e identificar las probables fuentes de compradores de la goma de huizache teniendo como base el uso de la goma arábiga.

\section{Planteamiento del problema}

Debido a la demanda de goma arábiga (Acacia senegal y Acacia seyal) en la industrias alimentaria, farmacéutica e industrial la cual puede ser sustituida por la de mezquite y la de huizache por la similitud en sus componentes. Se plantea el proyecto de producción de goma de huizache utilizando como inductor el etileno y analizando que el árbol no tenga algún daño biológico manifestado externamente.

\section{Justificación}

La FCAyF atendiendo el compromiso con los habitantes del desierto de Chihuahua, en el desarrollo en su línea de investigación, generación y transferencia de tecnología, la investigación aplicada para la producción de goma de mezquite y huizache en dicho Ejido, cuenta con 1500 ha de tierras ejidales en las cuales se distribuye de forma natural la especie de A. farnesiana y Prosopis spp que es objeto de estudio para calcular el potencial de producción de goma. Las cuales no se están aprovechando económicamente, por lo que se generó esta propuesta para generar economía y aprovechar el recurso. Se establece el método de producción y cosecha para las poblaciones nativas y plantaciones.

México cuenta con una variedad muy amplia de flora de la cual se extraen resinas y gomas que son utilizadas en distintas industrias. Algunas especias de acacias tienen la capacidad de producir goma y en su composición química llevan los carbohidratos y proteínas, dependiendo de la especie la proporción varia en estas (Verbeken et al., 2003). El huizache es utilizado como fijador de metales pesados en algunas regiones del país, es uno de los usos que se le puede dar al huizache (Landeros et al., 2011). Según Verbeken et al., 2003, la composición y las características bioquímicas de las gomas depende de su edad vegetal, del sitio donde se presenta la secreción, de las características del suelo y las condiciones climáticas donde se encuentra la planta. Estos son factores importantes para la producción de goma.

Usos: se cultiva como ornamental y por su leña. Es cultivada o fomentada como forrajera y para el control de erosión, sobre todo en suelos degradados. Es medicinal y se usa para curtir. Es la fuente de un aceite usado en la perfumería. (Márquez et al., 1999). La goma arábiga ha sido utilizada desde hace cientos de años en la industria alimenticia, en la farmacéutica, en vitivinícola, pinturas y recubrimientos además de la refresquera, papel y adhesivos, entre otros (Cabañas, 2012). Existen otras plantas que producen goma también. En México existen diversas especies del género acacia que pueden ser sustitutos de esta goma, sin embargo, en zonas semidesérticas se encuentra de forma natural la especie de Acacia farnesiana (huizache), la cual también produce secreciones semejantes a las de la goma arábiga. El huizache por su gran habilidad de adaptarse a varios tipos de suelo y clima cuenta con una amplia biodisponibilidad (Barrientos et al, 2012), en gran parte del estado de Chihuahua, sin embargo, no se ha logrado aprovechar de manera adecuada.

\section{MATERIALES Y MÉTODOS}

\section{Localización del área de estudio}

El área de trabajo está localizada en el ejido de Naica, en el municipio de Saucillo. Es una de las principales poblaciones núcleo dentro del municipio de Saucillo.

El otro sitio fue una plantación urbana de huizache de una ha en ciudad Delicias. Está situado en la zona central del estado de Chihuahua. El tipo de investigación fue descriptiva y correlacional. Las variables que se evaluaron fueron: la concentración de la hormona para determinar las 3 clases de tratamiento y el peso de la goma exudado por tratamiento. Se llevó a cabo de la siguiente manera: 
1. Se aplicó Etileno (Etephon), regulador de crecimiento líquido con una formula, Acido 2, (cloroetil) fosfórico; no menos de $21.70 \%$ en peso.

2. En la población nativa se realizó un estudio exploratorio para determinar ergonómicamente la altura de aplicación del tratamiento en el tronco principal. Se utilizaron alturas de 50, $60 \mathrm{y}$ $70 \mathrm{~cm}$, la longitud de corte de aplicación fue de $2.0,3.5$ y $5.0 \mathrm{~cm}$ y la profundidad del corte a $0.25,0.40$ y $0.60 \mathrm{~cm}$.

3. Con los resultados obtenidos se realizó un diseño experimental de bloques completamente al azar con tres dosis y 6 repeticiones en la población nativa los tratamientos fueron del $10 \%, 15 \%$ y el $20 \%$.

4. En la plantación urbana se realizó un diseño de bloques completamente al azar con los tratamientos del $10 \%, 15 \%$ y el $20 \%$ con 10 repeticiones cada uno.

5. Las variables de estudio son: la cantidad de goma exudada o escurrida por árbol (de tronco principal y observaciones del follaje y de los cortes.

6. El producto se aplicó con una jeringa en el corte.

7. Se usa un formón o cincel para remover la goma de la planta.

8. Posteriormente se pesó el exudado por árbol.

9. Algunas de las muestras se mandaron a un laboratorio químico, para conocer sus propiedades y determinar la similitud con las demás gomas.

10. Se utilizó el programa de SPSS IBM v22 para el análisis estadístico de las variables.

11. Se realizó un análisis proximal a las muestras de la goma de mezquite al $10 \% 15 \%$ y $20 \%$ y huizache recolectadas en las áreas nativas por el laboratorio de análisis de agua y alimentos de la UACH (REG EF/573, S.S.A. REG JMA-PSAL002-93. CED. PROF. 7915859).

\section{RESULTADOS}

Para cumplir con el primer objetivo, se hizo una selección de los árboles al azar de diferentes diámetros. Posteriormente se procedió a aplicar la hormona, para lo cual se procedió a realizar una incisión en el fuste o tronco principal. La altura de corte fue diferente en cada árbol siendo la altura, desde un punto de vista ergonómico, la que facilito la aplicación de la hormona y la recolección de la goma. Siendo esta, a partir de los $50 \mathrm{~cm}$ de altura del tronco principal. Los árboles seleccionados cumplieron con una característica común del árbol de huizache que fue el de tener tres o más ramas secundarias. La incisión de aplicación de la hormona se realizó considerando la parte anterior de la rama que tira al centro del árbol y no aplicarlo abajo porque se tira el producto y la goma se cae. El ángulo de corte fue entre $30^{\circ}$ y $40^{\circ}$ cuidando que la solución fuera absorbida por el árbol. La longitud óptima de corte fue de 2.5 centímetros. En virtud de que la corteza del árbol de huizache es más delgada, la profundidad de corte óptima fue de 0.5 centímetros de profundidad. Posteriormente se aplicó, en cada corte o incisión, el producto con una jeringa, en tres concentraciones: $15 \%, 20 \%$ y $25 \%$. Se utilizo una máscara para evitar absorber los vapores del producto y guantes para evitar el contacto con el mismo.

El tiempo en que la hormona fue absorbida y translocada fue de alrededor de ocho días en la plantación y de 15 en la nativa para recolectar o realizar la primera cosecha. Se utilizó el formón para despegar la goma y se colocó en bolsas plásticas una por árbol. Las posteriores recolecciones fueron cada siete días. Se pesaron y se registraron los pesos para realizar el análisis de los datos. Se realizó una comparación entre los diferentes tratamientos con la obtención de goma por árbol. La producción de goma en la población nativa fue de dos meses en tanto que en la plantación urbana fue de dos y medio meses.

A continuación, se presenta la tabla 1, correspondiente al experimento exploratorio con 21 árboles respectivamente, donde se arrojan los primeros resultados. 
Tabla 1. Resultados de tres porcentajes diferentes de tratamientos en el primer experimento aplicados el 28/04/2018. Población nativa.

\begin{tabular}{|c|c|c|c|c|c|c|c|c|}
\hline Árbol & Tratamiento & $\begin{array}{c}\text { Peso } \\
\text { goma 1R } \\
\text { gr. }\end{array}$ & $\begin{array}{c}\text { Peso } \\
\text { goma } 2 R \\
\text { gr. }\end{array}$ & $\begin{array}{c}\text { Peso } \\
\text { goma 3R } \\
\text { gr. }\end{array}$ & $\begin{array}{c}\text { Peso } \\
\text { goma 4R } \\
\text { gr. }\end{array}$ & $\begin{array}{c}\text { Peso } \\
\text { goma } \\
\text { total por } \\
\text { árbol gr. }\end{array}$ & $\begin{array}{c}\text { Peso promedio } \\
\text { por } \\
\text { árbol/tratamiento } \\
\text { gr. }\end{array}$ & $\begin{array}{c}\text { Producción } \\
\text { por ha= } \\
100 \text { árboles } \\
\text { kg. }\end{array}$ \\
\hline 1 & $10 \%$ & 37.34 & 45.78 & 18.87 & 34.00 & 135.99 & & \\
\hline 4 & $10 \%$ & 42.33 & 21.73 & 20.02 & 28.03 & 112.11 & & \\
\hline 7 & $10 \%$ & 26.65 & 81.44 & 27.46 & 45.18 & 180.73 & & \\
\hline 10 & $10 \%$ & 31.55 & 28.47 & 10.11 & 23.38 & 93.51 & & \\
\hline 13 & $10 \%$ & 0.57 & 12.90 & 7.16 & 6.88 & 27.51 & & \\
\hline 16 & $10 \%$ & 9.68 & 28.05 & 20.02 & 19.25 & 77.00 & 104.47 & 10.45 \\
\hline 2 & $15 \%$ & 125.37 & 61.91 & 15.45 & 67.58 & 270.31 & & \\
\hline 5 & $15 \%$ & 32.18 & 122.18 & 50.86 & 68.41 & 273.63 & & \\
\hline 8 & $15 \%$ & 22.86 & 62.17 & 7.76 & 30.93 & 123.72 & & \\
\hline 11 & $15 \%$ & 52.20 & 82.82 & 12.71 & 49.24 & 196.97 & & \\
\hline 14 & $15 \%$ & 2.03 & 44.12 & 19.12 & 21.76 & 87.03 & & \\
\hline 17 & $15 \%$ & 59.76 & 67.87 & 51.84 & 59.82 & 239.29 & 198.49 & 19.85 \\
\hline 3 & $20 \%$ & 65.51 & 52.90 & 9.21 & 42.54 & 170.16 & & \\
\hline 6 & $20 \%$ & 57.60 & 63.20 & 6.82 & 42.54 & 170.16 & & \\
\hline 9 & $20 \%$ & 128.09 & 139.65 & 18.52 & 95.42 & 381.68 & & \\
\hline 12 & $20 \%$ & 75.01 & 165.95 & 32.16 & 91.04 & 364.16 & & \\
\hline 15 & $20 \%$ & 3.00 & 64.97 & 35.15 & 34.37 & 137.49 & & \\
\hline 18 & $20 \%$ & 2.73 & 68.34 & 37.00 & 36.02 & 144.09 & 227.96 & 22.80 \\
\hline
\end{tabular}

Fuente: Elaboración propia con datos tomados en campo. 1R= Primera recolección.

En la tabla 2, se muestran los resultados del experimento que se aplicó a 18 árboles de huizache, de los cuales 6 fueron con un tratamiento del 10\%, 6 con el $15 \%$ y 6 con el $20 \%$. El mejor tratamiento fue el del 20\% con una producción promedio por árbol de 227.96 gr. y una producción estimada por hectárea de $22.80 \mathrm{~kg}$.

Tabla 2. Resultados de tres tratamientos en el experimento en plantación urbana de huizache, 2019.

\begin{tabular}{|c|c|c|c|c|c|c|c|c|}
\hline $\begin{array}{c}\text { Número } \\
\text { de árbol }\end{array}$ & Tratamiento & $\begin{array}{c}\text { 1ra } \\
\text { Cosecha } \\
\text { gr. }\end{array}$ & $\begin{array}{c}\text { 2da. } \\
\text { Cosecha } \\
\text { gr. }\end{array}$ & $\begin{array}{c}\text { 3ra. } \\
\text { Cosecha } \\
\text { gr. }\end{array}$ & $\begin{array}{c}\text { 4ta. } \\
\text { Cosecha } \\
\text { gr. }\end{array}$ & $\begin{array}{c}\text { Total } \\
\text { gr. }\end{array}$ & $\begin{array}{c}\text { Promedio } \\
\text { gr. }\end{array}$ & $\begin{array}{c}\text { Producción } \\
\text { ha= 625 } \\
\text { árboles kg. }\end{array}$ \\
\hline 1 & $10 \%$ & 102 & 64 & 44 & 70.07 & 280.27 & & \\
\hline 2 & $10 \%$ & 86 & 62 & 47 & 64.93 & 259.73 & \\
\hline
\end{tabular}


Magaña Magaña et al., 2019

\begin{tabular}{|c|c|c|c|c|c|c|c|c|}
\hline 3 & $10 \%$ & 116 & 108 & 94 & 105.87 & 423.47 & & \\
\hline 4 & $10 \%$ & 290 & 218 & 138 & 215.27 & 861.07 & & \\
\hline 5 & $10 \%$ & 230 & 210 & 130 & 190.00 & 760.00 & & \\
\hline 6 & $10 \%$ & 136 & 114 & 52 & 100.67 & 402.67 & & \\
\hline 7 & $10 \%$ & 48 & 42 & 23 & 37.80 & 151.20 & & \\
\hline 8 & $10 \%$ & 204 & 98 & 44 & 115.40 & 461.60 & & \\
\hline 9 & $10 \%$ & 266 & 226 & 151 & 214.27 & 857.07 & & \\
\hline 10 & $10 \%$ & 202 & 116 & 81 & 132.87 & 531.47 & 498.9 & 311.8 \\
\hline 1 & $15 \%$ & 32 & 14 & 12 & 19.33 & 77.33 & & \\
\hline 2 & $15 \%$ & 24 & 58 & 34 & 38.67 & 154.67 & & \\
\hline 3 & $15 \%$ & 50 & 52 & 16 & 39.33 & 157.33 & & \\
\hline 4 & $15 \%$ & 30 & 50 & 24 & 34.67 & 138.67 & & \\
\hline 5 & $15 \%$ & 44 & 66 & 34 & 48.00 & 192.00 & & \\
\hline 6 & $15 \%$ & 28 & 88 & 32 & 49.33 & 197.33 & & \\
\hline 7 & $15 \%$ & 10 & 26 & 16 & 17.33 & 69.33 & & \\
\hline 8 & $15 \%$ & 32 & 114 & 50 & 65.33 & 261.33 & & \\
\hline 9 & $15 \%$ & 20 & 36 & 18 & 24.67 & 98.67 & & \\
\hline 10 & $15 \%$ & 52 & 80 & 16 & 49.33 & 197.33 & 154.4 & 96.5 \\
\hline 1 & $20 \%$ & 4 & 60 & 44 & 36.00 & 144.00 & & \\
\hline 2 & $20 \%$ & 96 & 178 & 118 & 120.00 & 512.00 & & \\
\hline 3 & $20 \%$ & 22 & 42 & 20 & 28.00 & 112.00 & & \\
\hline 4 & $20 \%$ & 142 & 132 & 56 & 110.00 & 440.00 & & \\
\hline 5 & $20 \%$ & 62 & 128 & 92 & 94.00 & 376.00 & & \\
\hline 6 & $20 \%$ & 44 & 60 & 48 & 50.67 & 202.67 & & \\
\hline 7 & $20 \%$ & 48 & 68 & 18 & 44.67 & 178.67 & & \\
\hline 8 & $20 \%$ & 14 & 58 & 44 & 38.67 & 154.67 & & \\
\hline 9 & $20 \%$ & 46 & 76 & 18 & 46.67 & 186.67 & & \\
\hline 10 & $20 \%$ & 10 & 36 & 32 & 26.00 & 104.00 & 241.1 & 150.7 \\
\hline
\end{tabular}

Fuente: Elaboración propia con datos tomados en campo.

En la tabla 3, se presentan las producciones promedio por tratamiento en población nativa y plantación, lo que hace referencia a que la reacción de la hormona se manifiesta totalmente diferente en cada población. Se observa en base a la producción promedio que en la plantación se produjo mayor cantidad de goma para el porcentaje del $10 \%$, así mismo el tratamiento del $20 \%$ fue el de mayor producción en la población nativa.

Tabla 3. Comparativo de producción promedio por hectárea en población nativa versus plantación urbana.

\begin{tabular}{|c|c|c|}
\hline Tratamiento & $\begin{array}{c}\text { Producción promedio } \\
\text { población nativa kgrs. }\end{array}$ & $\begin{array}{c}\text { Producción promedio } \\
\text { plantación urbana kgrs. }\end{array}$ \\
\hline
\end{tabular}


Magaña Magaña et al., 2019

\begin{tabular}{|c|c|c|}
\hline $10 \%$ & 10.45 & 311.80 \\
\hline $15 \%$ & 19.85 & 96.50 \\
\hline $20 \%$ & 22.80 & 150.70 \\
\hline
\end{tabular}

Fuente: Elaboración propia con datos tomados en campo.

Para dar respuesta al objetivo 3 de esta investigación, se realizó el análisis proximal a las muestras de la goma de mezquite con los tratamientos del $15 \%$ y $20 \%$ recolectadas en las áreas nativas y una de huizache. Esta prueba fue realizada por el laboratorio de análisis de agua y alimentos de la UACH (REG EF/573, S.S.A. REG JMA-PSAL-002-93. CED. PROF. 7915859), el 26 de junio de 2018. El análisis químico proximal mostro diferencias en el contenido de los carbohidratos y proteínas de ambas especies de acacias (tabla 4).

La goma de huizache presento el contenido menor de carbohidratos (68.49\%), este valor es aproximadamente un $20 \%$ menor que en la goma de mezquite al $15 \%(80.69 \%)$ y que en la goma de mezquite al $20 \%$ en comparación de la goma arábiga que se está tomando de referencia y no se reportó este dato.

Tabla 4. Datos fisicoquímicos de las gomas analizadas comparadas con la goma arábiga.

\begin{tabular}{|l|l|l|l|l|l|}
\hline Especies & $\begin{array}{l}\text { Humedad } \\
\text { \% }\end{array}$ & $\begin{array}{l}\text { Ceniza } \\
\text { s \% }\end{array}$ & $\begin{array}{l}\text { Proteína } \\
\text { \% }\end{array}$ & $\begin{array}{l}\text { Grasa } \\
\text { total \% }\end{array}$ & $\begin{array}{l}\text { Carbohidrat } \\
\text { os \% }\end{array}$ \\
\hline $\begin{array}{l}\text { Goma de Huizache de } \\
\text { Naica (acacia } \\
\text { farnesiana) } 10 \%\end{array}$ & 17.49 & 4.37 & 5.09 & 4.53 & 68.49 \\
\hline $\begin{array}{l}\text { Goma de Mezquite de } \\
\text { Naica prosopis } \\
\text { glandulosa) (15\%) }\end{array}$ & 10.09 & 2.16 & 2.94 & 4.10 & 80.69 \\
\hline $\begin{array}{l}\text { Goma de Mezquite de } \\
\text { Naica prosopis } \\
\text { glandulosa) (20\%) }\end{array}$ & 10.49 & 2.19 & 6.65 & 6.40 & 74.26 \\
\hline $\begin{array}{l}\text { Goma Arábiga (acacia } \\
\text { senegal y acacia seyal) }\end{array}$ & $10-20$ & 5 & 2.1 & $\mathrm{NR}$ & $\mathrm{NR}$ \\
\hline
\end{tabular}

Fuente: Elaboración propia con datos de laboratorio de la Facultad de Ciencias Químicas de la UACH y Reséndiz et al.

(2016).

Los carbohidratos son un importante componente químico que modifica la capacidad de espesamiento y promueve la estabilidad de emulsiones de agua (Adewusi et al., 2010). La fracción que presentan las gomas de carbohidratos juegan un papel muy importante en la estabilización, ya que esta fracción está asociada con el impedimento estérico entre las gotas de la emulsión, las cuales reducen y evitan la coalescencia de gotas por contacto (Gulrez, et al. 2011).

Los altos contenidos de proteína están relacionados con la capacidad emulsionante. La goma de mezquite al (20\%) mostro el mayor contenido de proteína (6.65\%) que es 2 veces mayor que las otras gomas. Este valor es mayor que el contenido de proteína registrado para Paolii $0.34 \%$ (Anderson \& Weiping, 1990). Así mismo, la acacia polyacantha tuvo un contenido de $2.9 \%$ (S. Mhinzi \& Mrosso.1995).

Algunos autores como Wang et al. (2011) menciona que las proteínas tienen una actividad superficial e interacciones electrostáticas que promueven la formación, engrosamiento y estabilización de la emulsión. Por lo que la proporción carbohidrato/proteína de la goma podría ser responsable de la estabilidad de las emulsiones. 
La goma de huizache tuvo el contenido de ceniza mayor (4.37\%) que la goma de Mezquite al 15\% $(2.16 \%)$ a comparación con la referencia en donde estándares mínimos para la goma arábiga de buena calidad han sido definidos en la Farmacopea de los Estados Unidos, Edición XVII (1965) de la siguiente forma: $4 \%$ de cenizas totales (máximo), $0.5 \%$ de cenizas insolubles al ácido (máximo), $1 \%$ de residuo insoluble en agua (máximo).
Mientras que el contenido de humedad de la goma de Huizache fue mucho mayor $(17.49 \%)$ que las otras dos gomas. El contenido de grasas totales fue muy similar en todas las gomas analizadas.

Así mismo, se realizó la comparación de todos los parámetros del análisis proximal de las gomas de mezquite al $15 \%$ y $20 \%$ y huizache de las muestras recolectadas en Naica, Saucillo, Chihuahua con los resultados de Reséndiz, et al. (2016), como observan en la tabla 5 .

Tabla 5. Comparación de análisis proximal de la goma de mezquite con tratamiento aplicado al $15 \%$ y $20 \%$ y huizache en Naica, Chihuahua (2018) versus resultados de Reséndiz, et al. (2016) en goma de mezquite, huizache y goma arábiga.

\begin{tabular}{|l|l|l|l|l|l|l|}
\hline Parámetros & $\begin{array}{l}\text { Mezquite } \\
\text { tratamiento } \\
\mathbf{1 5 \%} \\
\text { Naica 2018 }\end{array}$ & $\begin{array}{l}\text { Mezquite } \\
\text { tratamiento } \\
\mathbf{2 0 \%} \\
\text { Naica 2018 }\end{array}$ & $\begin{array}{l}\text { Mezquite } \\
\text { Plantación } \\
\text { Reséndiz, } \\
\text { et al. 2016 }\end{array}$ & $\begin{array}{l}\text { Huizache } \\
\text { Naica 2018 }\end{array}$ & $\begin{array}{l}\text { Huizache } \\
\text { Plantación } \\
\text { Reséndiz, } \\
\text { et al. 2016 }\end{array}$ & $\begin{array}{l}\text { Goma } \\
\text { Arábiga } \\
\text { Reséndiz, } \\
\text { et al. 2016 }\end{array}$ \\
\hline Humedad \% & 10.09 & 10.49 & 10.25 & 17.49 & 12.65 & 10 a 20 \\
\hline Cenizas \% & 2.16 & 2.19 & 2.63 & 4.37 & 3.69 & 5 \\
\hline Proteína \% & 2.94 & 6.65 & 4.72 & 5.09 & 7.94 & $2.1+-0.2$ \\
\hline Grasa total \% & 4.10 & 6.40 & NR & 4.53 & NR & NR \\
\hline $\begin{array}{l}\text { Carbohidratos } \\
\%\end{array}$ & 80.69 & 74.26 & 5.96 & 68.49 & 2.2 & NR \\
\hline
\end{tabular}

Fuente: Elaboración propia con datos del laboratorio de análisis de agua y alimentos de la UACH y de Reséndiz, et al. (2016). NR = No reportado.

Los resultados que se obtuvieron en el análisis proximal de las muestras de goma de mezquite obtenidas en Naica Chihuahua en el año 2018, se observan en el cuadro 6 y se compararon con los del estudio realizado en Celaya, Guanajuato por Reséndiz et al. (2016) en goma de mezquite, huizache y goma arábiga.

$\mathrm{Al}$ respecto las muestras de mezquite y huizache de Naica, Chihuahua dieron porcentajes de humedad muy similares a los obtenidos en Celaya, Guanajuato comparadas con la de la goma arábiga que observa un rango que va de $10 \%$ al $20 \%$, en dicho rango se encuentran todas las muestras en el entendido que en el límite inferior del $10 \%$ es el límite mínimo aceptable, en tanto el límite máximo de la goma arábiga que es el máximo aceptable. Esto es muy importante en virtud de que la humedad puede ser un factor con una ilusión óptica en el peso cuando su humedad es mayor. El contenido de cenizas de la goma arábiga es del 5\%. Las muestras de la goma de mezquite de Naica, Chihuahua y Celaya, Guanajuato en relación a este parámetro, se encuentran en un rango de $2.16 \%$ a $2.63 \%$ que están por debajo del porcentaje de cenizas de la goma arábiga. En tanto que la goma de huizache de Naica, Chihuahua es la más cercano a la goma arábiga.

El contenido de proteína más alto fue el de la goma de huizache de Celaya, Guanajuato con $7.94 \%$. Siguiéndole en ese orden la goma de mezquite con el tratamiento del 20\%. Comparando los contenidos de proteína, encontramos que el porcentaje de la goma arábiga, está por debajo de todos los porcentajes de las gomas de mezquite y huizache. En el contenido de grasa total predominan el del mezquite con tratamiento al $20 \%$ y el huizache, obtenidas en Naica, Chihuahua. Los porcentajes de 
contenido de carbohidratos en mezquite y huizache de Naica, Chihuahua son mayores por encima de los obtenidos en las muestras de Celaya, Guanajuato sin poder compararse con la goma arábiga la cual no se reportó en este informe.

Así mismo dando respuesta al objetivo 4, se establecieron las fuentes de compradores para su comercialización. Entre ellos se encuentran: BAFAR, Industrias Coca Cola, Dulces Montes, De la Rosa, Dulces Benny, Chocolates Ibarra, Cosméticos Natura, JAFRA, entre otros.

\section{CONCLUSIONES}

Se comprobó mediante el análisis químico proximal que ambas gomas de mezquite y huizache tienen semejanza e incluso mejora los parámetros requeridos por la Food and Drug Administration para el uso de la goma arábiga en la industria alimenticia. La goma de estas dos especies es un buen sustituto de la goma arábiga presentando un potencial similar e incluso mayor. Lo que concuerda con lo citado por López-Franco et al., 2006 donde menciona que las gomas del mezquite y huizache, pueden ser un insumo de las industrias cosmética, medicinal y alimenticia, y podrían reducir la alta demanda que tiene la goma arábiga, presentando una alternativa de uso industrial.

Los resultados arrojaron que los árboles de huizache nativos a los que se les aplico el tratamiento con mayor concentración (20\%) son los que presentan mayor producción de goma. En tanto que en la plantación urbana el tratamiento que dio mayor producción de goma fue el menor (10\%). Haciendo una prospección en las plantaciones nativas se consideró una producción por hectárea de $22.80 \mathrm{~kg}$, en tanto que la producción promedio estimada en la plantación urbana fue de $311.80 \mathrm{~kg}$. Los árboles tanto en la población nativa como en la urbana, no manifestaron signos de deterioro.

\section{RECOMENDACIONES}

- Hacer un plan de manejo en la población nativa para mejorar la constitución fisiológica del árbol dándole espacios de cinco metros entre planta y planta.

- Establecer plantaciones nativas con plantas derivadas de árboles de huizache seleccionados por su producción de goma.

- Establecer plantaciones regadas con aguas tratadas para aprovechar que le huizache es un recolector de metales y mejorador de suelos.

- Elaborar un proyecto de inversión para financiar la creación de un laboratorio para purificar la goma.

- Por último, es necesario crear una empresa cooperativa para comercializar la goma de huizache y del mezquite.

\section{LITERATURA CITADA}

Adewusi E, Afolayan A. (2010). A review of natural products with hepatoprotective activity. J Medicinal Plants Res.;4(13):1318-34.

Anderson, D.M.W. \& Weiping, Wang. (1990). The characterization of Acacia paolii gum and four commercial Acacia gums from Kenya. Food Hydrocolloids. 3. 475-484. https://doi.org/10.1016/S0268005X(09)80225-7

Barrientos-Ramírez, Lucía; Vargas-Radillo, J. Jesús; Rodríguez-Rivas, Antonio; Ochoa-Ruíz, Héctor Guillermo; Navarro-Arzate, Fernando; Zorrilla, José Evaluación de las características del fruto de huizache (Acacia farnesiana (L.) Willd.) para su posible uso en curtiduría o alimentación animal Madera y Bosques, vol. 18, núm. 3, 2012, pp. 2335 Instituto de Ecología, A.C. Xalapa, México https://doi.org/10.21829/myb.20

Cabañas G., E. 2012. Caracterización Bioquímica y Propiedades Viscoelásticas de Gomas de Acacia farnesiana (L.) Willd. y Acacia cochliacantha Humb. \& 
Bonpl. ex Willd. Tesis maestría. Maestro en Ciencias en Desarrollo de Productos Bióticos, Morelos, México. 51p.

Conferencia de la Naciones Unidas sobre comercio y desarrollo, 2018. Goma arábiga: el aumento de la demanda crea nuevas oportunidades para los productores africanos.

Desierto

Chihuahuense.http://www.wwf.org.mx/ que_hacemos/programas/desierto_chihu ahuense

Google maps. Disponible en: https://www.google.com/maps

Gulrez, Syed \& Al-Assaf, Saphwan \& Phillips, Glyn. (2011). Hydrogels: Methods of preparation, Characterisation and Applications. https://doi.org/10.5772/24

INEGIhttp://www.inegi.org.mx/est/contenidos/ espanol/metodologias/censos/cepafop/de fault.asp?c $=545$

Landeros-Márquez, et al (2011). Uso potencial del huizache (Acacia farnesiana L. Will) en la fitorremediación de suelos contaminados con plomo. Rev. Chapingo serie ciencias forestales y del ambiente vol.17 no.spe Chapingo ene. 2011 https://doi.org/10.5154/r.rchscfa.2010.08

López-Franco, Yolanda L.; Goycoolea, Francisco M.; Valdez, Miguel A.; Calderón de la Barca, Ana María, Goma de mezquite: una alternativa de uso industrial Interciencia, vol. 31, núm. 3, marzo, 2006, pp. 183-189 Asociación Interciencia Caracas, Venezuela.

Márquez, A. C., F. Lara O., B. Esquivel R. y R. Mata E. 1999. Plantas medicinales de México II. Composición, usos y actividad biológica. Universidad Nacional Autónoma de México. México, D. F.
Reséndiz N. et al. (2016) Goma de mezquite y huizache como alternativa de aprovechamiento en sistemas agroforestales. Revista Mexicana de Ciencias Agrícolas, núm. 16, mayojunio, 2016, pp. 3251-3261 Instituto Nacional de Investigaciones Forestales, Agrícolas y Pecuarias. Estado de México, México https://doi.org/10.29312 remexca.v0i16.394

Rzedowski, G. C. y J. Rzedowski (eds.). Flora del Bajío y de regiones adyacentes. Fascículo 150. Instituto de EcologíaCentro Regional del Bajío. Consejo Nacional de Ciencia y Tecnología y Comisión Nacional para el Conocimiento y Uso de la Biodiversidad. Pátzcuaro, Michoacán, México.

S. Mhinzi, G \& Mrosso, Hillary. (1995). Studies on Tanzanian Acacia gums. Part 3. Some properties of gum exudates from the series Vulgares and Gummiferae. Food Chemistry - FOOD CHEM. 54. 261-264. https://doi.org/10.1016/0308-8146(95)0 0038-K

Sutton, A. 2000. El Desierto Chihuahuense, nuestro desierto. Fondo Mundial para la Naturaleza.

URL: http://www.pronatura.org.

Verbeken, D., Dierckx S. \& Dewettinck K. 2003. Exudate gums: ocurrence, production, and applications. Applied Microbiology and Biotechnology, 63: $10-21$.

https://doi.org/10.1007/s00253-003-1354 $-\mathrm{Z}$

Wang L, et al. (2011) Growth propagation of yeast in linear arrays of microfluidic chambers over many generations. Biomicrofluidics 5(4):44118-441189. https://doi.org/10.1063/1.3668243 
Copyright (c) 2019 José Eduardo Magaña Magaña, Maria de los Ángeles Romero Lara, Victor Hugo Villarreal Ramirez, Luisa Patricia Uranga V alencia y Christian Mauricio Kiessling Davison

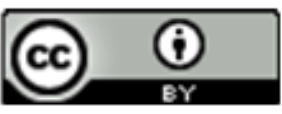

Este texto está protegido por una licencia licencia CreativeCommons 4.0

Usted es libre para Compartir —copiar y redistribuir el $\mathrm{m}$ aterial en cualquier medio o formato- $y$ Adaptar el documento - remezclar, transformar y crear a partir del material- para cualquier propósito, incluso para fines comerciales, siempre que cumpla la condición de:

Atribución: Usted debe dar crédito a la obra original de manera adecuada, proporcionar un enlace a la licencia, e indicar si se han realizado cambios. Puede hacerlo en cualquier forma razonable, pero no de forma tal que sugiera que tiene el apoyo del licenciante o 10 recibe por el uso que hace de la obra.

Resumendelicencia - Textocompletodelalicencia 